\title{
A THEOREM ON EXTENDING REPRESENTATIONS
}

\author{
RAYMOND C. FABEC ${ }^{1}$
}

\begin{abstract}
We obtain sufficient conditions for a unitary representation of a closed subgroup $M$ of a separable locally compact group $G$ to have a unique unitary extension to $G$. The conditions depend on the behavior of the representation on a closed normal subgroup $N$ of $G$ contained in $M$. We then discuss an application of the theorem in particle physics.
\end{abstract}

Let $N$ be a closed normal subgroup of a separable locally compact group $G$, and let $M$ be a closed subgroup of $G$ containing $N$. Let $H=\{m \in M$ : $g m g^{-1} \in M$ for $\left.g \in G\right\}$. Assume $L$ is a representation of $M$ such that $\left.L\right|_{N}$ is type I, and $\left.L\right|_{N}=\int_{N}^{\oplus} n(\alpha) \alpha d \mu(\alpha), \mu$ a standard measure on $\hat{N}$; i.e. there is a $\mu$ conull set in $\hat{N}$ which is standard. By the von Neumann selection theorem, there is a cross-section $c$ from $\hat{N}$ into the concrete irreducible representations of $N$ such that $c$ is Borel on a conull set in $\hat{N}$. Then

$$
\int_{\hat{N}}^{\oplus} n(\alpha) \alpha d \mu(\alpha)=\int_{\hat{N}}^{\oplus} n(\alpha) c(\alpha) d \mu(\alpha),
$$

and this gives the primary decomposition of $\left.L\right|_{N} \cdot G$ acts on $\hat{N}$ by $\alpha \cdot g=\{R$. $g: R \in \alpha\}$ where $(R \cdot g)(n)=R\left(g n g^{-1}\right)$. We do the transitive case first.

THEOREM 1. Assume $\mu$ is supported on $\alpha_{0}$. G. Assume the stabilizer $K$ of $\alpha_{0}$ is contained in $H$ and $\left.L\right|_{H} \cdot g$ is unitarily equivalent to $\left.L\right|_{H}$ for each $g \in G$. Then $L$ has a unique unitary extension to $G$. In fact, there is a representation $W$ of $K$ such that $\operatorname{ind}_{K}^{G} W$ is the extension. This extension is irreducible precisely when $W$ is irreducible.

REMARK. $L=\int_{G / M}^{\oplus} \operatorname{ind}_{x^{-1} K x}^{M} W \cdot x d \bar{\mu}(x)$ where $\bar{\mu}$ is a quasi-invariant measure on $G / M$.

Proof. Let $P$ be the projection valued measure based on the Borel subsets of $\alpha_{0} \cdot G \simeq K \backslash G$ giving the direct integral decomposition $\left.L\right|_{N}=$ $\int^{\oplus} n(\alpha) c(\alpha) d \mu(\alpha)$. Since $\left.L\right|_{H} \cdot g \simeq L$ for $g \in G,\left.\left.L\right|_{N} \cdot g \simeq L\right|_{N}$ for $g \in G$; and the argument given by Mackey [4, p. 296], shows $\mu$ is quasi-invariant and $n\left(\alpha \cdot g^{-1}\right)=n(\alpha)$ for $\mu$-a.e. $\alpha$ for each $g$. Hence, since $G$ acts transitively, $n$ is essentially constant. Therefore

$$
\left.L\right|_{N} \simeq n \int^{\oplus} c(\alpha) d \mu(\alpha)=n \int^{\oplus} c(\alpha) d \mu(\alpha)=n \int^{\oplus} \omega \cdot \gamma(K x) d \mu(K x)
$$

Received by the editors February 27, 1978 and, in revised form, July 6, 1978.

AMS (MOS) subject classifications (1970). Primary 22D10, 22D30; Secondary $22 E 70$.

'Research supported in part by a Louisiana State Summer Faculty Research Award. 
where $\omega \in \alpha_{0}$ and $\gamma: K \backslash G \rightarrow G$ is a Borel cross-section.

Since $\left.L_{m} L\right|_{N} L_{m}^{-1}=\left.\left.L\right|_{N} \cdot m \simeq L\right|_{N}$ and $n \int^{\oplus} \omega \cdot \gamma(K x) d \mu(K x)$ is the central decomposition of $\left.L\right|_{N}, L_{m} P(E) L_{m}^{-1}=P\left(E \cdot m^{-1}\right)$ for Borel subsets $E$ of $K \backslash G$ and $m \in M$. Furthermore, the orbit space $(K \backslash G)^{M}$ of $K \backslash G$ under $M$ is isomorphic to $G / M$. In fact $K x M=x x^{-1} K x M=x M$, for $x^{-1} K x \subset$ $x^{-1} H x=H$. Hence the orbit space is standard; and if $\bar{\mu}$ is the image of $\mu$ under the map $K x \mapsto x M, \mu$ decomposes uniquely $\bar{\mu}$-a.e. into an "integral of meaures", $\mu=\int_{G / M} \mu_{x M} d \bar{\mu}(x M)$, where each $\mu_{x M}$ is a Borel measure on $K \backslash G$ with $\mu_{x M}(K \backslash G-K x M)=0$ (see [2, Theorem 11]). Since $\mu$ is quasiinvariant under $M, \mu_{x M}$ is quasi-invariant under $M$ for $\bar{\mu}$-a.e. $x M$.

The decomposition $\mu=\int_{G / M} \mu_{x M} d \bar{\mu}(x M)$ yields a decomposition of $P$ into $\int_{G / M}^{\oplus} P(x M) d \bar{\mu}(x M)$ where each $P(x M)$ is a projection valued measure defined on the Borel subsets of $x M$ satisfying $P(x M)(E)=0$ iff $\mu_{x M}(E)=0$. Define a projection valued measure $Q$ on the Borel subsets of $G / M$ by $Q(F)=P(\cup F)$. Then

$$
L_{m}^{-1} Q(F) L_{m}=P(\bigcup F \cdot m)=P(\bigcup F)=Q(F) .
$$

Therefore $L$ decomposes into a direct integral over $G / M$; namely, $L=$ $\int^{\oplus} L(x M) d \bar{\mu}(x M)$. Furthermore,

$$
\left.L(x M)\right|_{N}=n \int_{K x M}^{\oplus} \omega \cdot \gamma(K z) d \mu_{x M}(K z)
$$

and

$$
L(x M)\left(m^{-1}\right) P(x M)(E) L(x M)(m)=P(x M)(E \cdot m),
$$

$E$ any Borel subset of $K x M$. Hence the pair $(L(x M), P(x M))$ forms a transitive system of imprimitivity based on the transitive $M$ space $K x M$. Let $\sigma: G / M \rightarrow G$ be a Borel cross-section. By Mackey's imprimitivity theorem [3, Theorem 6.6], $L(x M)=\operatorname{ind}_{\sigma(x M)^{-1} K \sigma(x M)} W(x M)$, where $W(x M)$ is a unitary representation of $\sigma(x M)^{-1} K \sigma(x M)$. One can show $\left.W(x M)\right|_{N} \simeq n \omega \cdot \sigma(x M)$.

We consider the representation $\left.L\right|_{H}$. The orbit space $(K \backslash G)^{H}$ of $K \backslash G$ under $H$ is isomorphic to $H \backslash G$ since $K x H=K x H x^{-1} x=H x$. Hence $\mu=$ $\int \nu_{H x} d \bar{\nu}(H x)$ where $\bar{\nu}$ is the image of $\mu$ under the map $K x \rightarrow H x$ and $\nu_{H x}$ is an $H$ quasi-invariant measure on $H x$. This decomposition, as before, gives a decomposition of $P$ into $\int^{\oplus} P(H x) d \bar{\nu}(H x)$.

$\bar{\mu}$ is the image of $\bar{\nu}$ under the map $H x \rightarrow x M$. Therefore $\bar{\nu}$ decomposes relative to $\bar{\mu}: \bar{\nu}=\int_{G / M} \bar{\nu}_{x M} d \bar{\mu}(x M), \bar{\nu}_{x M}$ a measure on $H \backslash G$ with

$$
\bar{\nu}_{x M}(H \backslash G-x M)=0 \text {. }
$$

Hence

$$
\begin{aligned}
\mu & =\int_{H \backslash G} \nu_{H x} d \bar{\nu}(H x)=\int_{G / M} \int_{H \backslash G} \nu_{H y} d \bar{\nu}_{x M}(H y) d \bar{\mu}(x M) \\
& =\int_{G / M} \mu_{x M} d \bar{\mu}(x M) .
\end{aligned}
$$


By uniqueness of decompositions, $\mu_{x M}=\int_{H \backslash G} \nu_{H y} d \bar{\nu}_{x M}(H y)$ for $\bar{\mu}$-a.e. $x M$. Furthermore, one has $P(x M)=\int_{H \backslash G} P(H y) d \bar{v}_{x M}(H y)$.

Define a projection valued measure $Q^{*}$ on the Borel subsets of $H \backslash G$ by $Q^{*}(F)=P(\cup F)$. Then $L_{h} Q^{*}(F) L_{h}^{-1}=Q^{*}(F)$ for $h \in H$. Therefore $\left.L\right|_{H}$ decomposes into a direct integral:

$$
\left.L\right|_{H}=\int_{H \backslash G}^{\oplus} L(H y) d \bar{\nu}(H y),
$$

where $\left.L(H y)\right|_{N}=n \int_{H y} \omega \cdot \gamma(K z) d \nu_{H y}(K z)$. Furthermore, since $P(x M)=$ $\int_{x M} P(H y) d \bar{\nu}_{x M}(H y)$, one has

$$
\left.L(x M)\right|_{H}=\left.\underset{\sigma(x M)^{-1}}{\operatorname{ind}} \underset{K \sigma(x M)}{M} W(x M)\right|_{H}=\int^{\oplus} L(H y) d \bar{\nu}_{x M}(H y) .
$$

This decomposition of $(L(x M), P(x M))$ is precisely the one given by Mackey's subgroup theorem. Note $K x M$ as an $M$ space is isomorphic to $\sigma(x M)^{-1} K \sigma(x M) \backslash M$ and the orbit space of $K x M$ under $H$ is isomorphic to the double coset space of $\sigma(x M)^{-1} K \sigma(x M)-H$ double cosets in $M$. The correspondence with $K x M$ carries the double coset $\sigma(x M)^{-1} K \sigma(x M) m H$ to $K \sigma(x M) m H=H \sigma(x M) m$. Hence the double cosets of $K x M$ are the $H$ cosets in $K x M$. Now let $\rho: H \backslash G \rightarrow G$ be a Borel cross-section. Then $\left.\rho\right|_{K x M} \rightarrow G$ is a cross-section of the $K-H$ double cosets in $K x M$ and $H y=$ $K \sigma(x M) \sigma(x M)^{-1} \rho(H y) H$. Therefore

$$
L(H y) \simeq \underset{\rho(H y)^{-1} K \rho(H y)}{\operatorname{H}} W(x M) \cdot \sigma(x M)^{-1} \rho(H y)
$$

$\bar{\nu}_{x M}$-a.e. $H y \bar{\mu}$-a.e. $x M$. For more details, see the proof of the subgroup theorem [4, p. 227].

We now have

$$
\left.L\right|_{H} \simeq \int_{H \backslash G}^{\oplus} \underset{\rho(H y)^{-1} K_{\rho}(H y)}{\operatorname{H}} W(y M) \cdot \sigma(y M)^{-1} \rho(H y) d \bar{\nu}(H y)
$$

and $P=\int^{\oplus} P(H y) d \bar{\nu}(H y)$ where $P(H y)$ is the canonical projection valued measure associated with the induced representation $L(H y)$. Therefore

$$
\left.\left.L\right|_{H} \simeq L\right|_{H^{\prime}} g \simeq \int_{g^{-1} \rho(H y)^{-1} K \rho(H y) g}^{i} W(y M) \cdot \sigma(y M)^{-1} \rho(H y) g d \bar{\nu}(H y) .
$$

Since $\rho(H y) g=\rho(H y g) h$ for some $h$,

$$
\left.L\right|_{H} \simeq \int^{\oplus} \underset{\rho(H y g)^{-1} K \rho(H y g)}{\operatorname{ind}_{i}} W(y M) \cdot \sigma(y M)^{-1} \rho(H y g) d \bar{\nu}(H y) .
$$

In this last direct integral decomposition, $P$ decomposes into $P=\int^{\oplus} P(H y)$. $g d \bar{\nu}(H y)$ where $P(H y) \cdot g(E)=P(H y)\left(E \cdot g^{-1}\right)$. Changing $y \rightarrow y g^{-1}$ in the last decomposition yields

$$
\left.L\right|_{H} \simeq \int^{\oplus} \underset{\rho(H y)^{-1} K \rho(H y)}{\operatorname{ind}} W\left(y g^{-1} M\right) \cdot \sigma\left(y g^{-1} M\right)^{-1} \rho(H y) d \bar{\nu}(H y)
$$


and

$$
P=\int^{\oplus} P\left(H y g^{-1}\right) \cdot g d \bar{\nu}(H y)
$$

But

$$
P\left(\mathrm{Hyg}^{-1}\right) \cdot \mathrm{g}=P(\mathrm{Hy}) .
$$

Hence we have two decompositions of $\left(\left.L\right|_{H}, P\right)$ relative to $Q^{*}$. Therefore

$$
\text { (ind } \begin{aligned}
W\left(y g^{-1} M\right) \cdot \sigma & \left.\left(y g^{-1} M\right)^{-1} \rho(H y), P(H y)\right) \\
& \simeq\left(\text { ind } W(y M) \cdot \sigma(y M)^{-1} \rho(H y), P(H y)\right)
\end{aligned}
$$

for $\bar{\nu}$-a.e. $H y$. It follows that

$$
W\left(y g^{-1} M\right) \cdot \sigma\left(y g^{-1} M\right) \rho(H y) \simeq W(y M) \cdot \sigma(y M)^{-1} \rho(H y)
$$

for $\bar{\nu}$-a.e. $H y$ for each $g \in G$. Hence there is a representation $W$ of $K$ such that $W(x M)$ is unitarily equivalent to the representation $\sigma(x M)^{-1} k \sigma(x M) \rightarrow$ $W(k)$ for $\bar{\mu}$-a.e. $x M$. We then have

$$
L \simeq \int_{G / M}^{\oplus} \underset{\sigma(x M)^{-1} K \sigma(x M)}{\operatorname{ind}} W \cdot \sigma(x M) d \bar{\mu}(x M) .
$$

Let $U=\operatorname{ind}_{K}^{G} W$. By Mackey's subgroup theorem, $U$ extends $L . U$ is unique for if $U$ is an extension of $L,(U, P)$ is a transitive system of imprimitivity based on $K \backslash G$. Hence $U=\operatorname{ind}_{K}^{G} W^{\prime}$. But then

$$
\begin{aligned}
\left(\underset{\sigma(x M)^{-1} K \sigma(x M)}{\operatorname{Min}} W\right. & \cdot \sigma(x M), P(x M)) \\
& \simeq\left(\underset{\sigma(x M)^{-1} K \sigma(x M)}{\operatorname{ind}} W^{\prime} \cdot \sigma(x M), P(x M)\right)
\end{aligned}
$$

for $\bar{\mu}$-a.e. $x M$. Hence $W \simeq W^{\prime}$.

The commuting rings of $U$ and $W$ are isomorphic. Hence $U$ is irreducible when $W$ is. Q.E.D.

REMARK. The stabilizer $K$ of $\alpha_{0}$ is contained in $H$ if the stabilizer of $\mu$-a.e. $\alpha$ is contained in $M$.

We next assume $\mu$ is based on a standard $G$-invariant subset $S$ of $\hat{N}$.

THEOREM 2. Assume $\mu$ is based on $S$ and $G$ acts regularly on $S$ and $\left.\left.L\right|_{H} \cdot g \simeq L\right|_{H}$ for $g \in G$. Then $L$ has a unique extension from $M$ to $G$ provided that the stabilizer of $\alpha$ is contained in $M$ for $\mu$-a.a. $\alpha$.

Proof. Let $S^{G}$ be the orbit space for $S$ under $G$. Let $\bar{\mu}$ be the quotient measure. The action is regular if there exists a $\bar{\mu}$ conull set $\bar{S}_{\underline{0}}$ in $S^{G}$ such that $\bar{S}_{0}$ is standard. Replacing $S$ by the inverse image $S_{0}$ of $\bar{S}_{0}$ in $S$, we may 
assume $S^{G}$ is standard. Let $P$ be the projection valued measure defined in the proof of Theorem 1. The measure $\mu$ decomposes relative to $\bar{\mu} ; \mu=\int \mu_{\bar{s}} d \bar{\mu}(\bar{s})$ where each $\mu_{\bar{s}}$ is a $G$ quasi-invariant measure based on $\bar{s}$. This leads to a decomposition of both $P$ and $L: P=\int^{\oplus} P^{\bar{s}} d \bar{\mu}(s)$ and $L=\int^{\oplus} L^{\bar{s}} d \bar{\mu}(\bar{s})$. Since the stabilizer of $\alpha$ is contained in $M$ for $\mu$-a.a. $\alpha \in S$, the stabilizer of $\alpha \in \bar{s}$ is contained in $M$ for $\mu_{s}$-a.a. $\alpha$ for $\bar{\mu}$-a.a. $\bar{s}$. Since $\left.\left.L\right|_{H} \cdot g \simeq L\right|_{H}$, $\left.\left.L^{\bar{s}}\right|_{H} \cdot g \simeq L^{\bar{s}}\right|_{H}$ for $\bar{\mu}$-a.e. $\bar{s}$. By Fubini's theorem, for $\bar{\mu}$-a.e. $\bar{s},\left.L^{\bar{s}}\right|_{H} \cdot g \simeq$ $\left.L^{\bar{s}}\right|_{H}$ a.e. $g$. But $\left\{g:\left.\left.L^{\bar{s}}\right|_{H} \cdot g \simeq L^{\bar{s}}\right|_{H}\right\}$ is a subgroup of $G$. Hence for $\bar{\mu}$-a.e. $\bar{s}$, $\left.L^{s}\right|_{H}$ is invariant under $G$. Hence we may apply Theorem 1 and obtain an extension $R^{\bar{s}}$ of $L^{\bar{s}}$ to $G$ for $\bar{\mu}$-a.a. $\bar{s}$. The extension is a Borel function of $\bar{s}$ and hence $\int^{\oplus} R^{s} d \bar{\mu}(\bar{s})$ is an extension of $L$. Since $R^{\bar{s}}$ is unique a.e. $\bar{s}$, this extension is unique. Q.E.D.

REMARK. These theorems remain valid for $\sigma$-representations. One can either work in the group extension or modify the above proofs.

As an application of the theorem we show how it is possible to determine the dynamics of a free particle system having time translations which do not commute with spatial translations. For instance the spatial transformations of $\mathbf{R}^{3}$ may be the Euclidean group $E_{3}=\left\{(x, R): x \in \mathbf{R}^{3}\right.$ and $\left.R \in \mathrm{SO}(3)\right\}$ with multiplication defined by $(x, R)(y, S)=(x+R y, R S)$. Time translation by $t$, however, will have the property $t(x, R)=\left(e^{t} x, R\right) t$. This may occur, for instance, if physical space expands exponentially with time. In any case, for this group a free particle of spin $j, j$ a half integer, is a projective representation $U$ such that $U_{(x, R)} f(y)=e^{2 \pi i x \cdot y} D^{j}(R) f\left(R^{-1} y\right)$ for $f \in L^{2}\left(\mathbf{R}^{3}, C^{2 j+1}\right)$ where $D^{j}$ is the projective irreducible representation of $\mathrm{SO}(3)$ with dimension $2 j+1$. We shall, for convenience, assume $j$ is an integer, for in this case $D^{j}$ is an ordinary representation. Next note one has $M=E_{3}$ and $N=\mathbf{R}^{3}$. Then

$$
\left.U\right|_{\mathbf{R}^{3}}=(2 j+1) \int_{\mathbf{R}^{3}}^{\oplus} e^{2 \pi i x \cdot()} d x
$$

and since

$$
\exp (2 \pi i x \cdot()) \cdot t(0, R)=\exp \left(2 \pi i e^{-t} R^{-1} x \cdot()\right),
$$

the orbit of $e_{1}=(1,0,0)$ is almost all of $\mathbf{R}^{3}$. The stabilizer $K$ of $e_{1}$ is $\{(y, R)$ : $\left.R e_{1}=e_{1}, y \in \mathbf{R}^{3}\right\}$ while $H=M=E_{3}$. Hence Theorem 1 applies and we need only find $W$. Note $\left.U\right|_{E_{3}}=\int_{\mathbf{R}}^{\oplus} W(r) d r$ where $W(r)$ is the representation of $E_{3}$ on $L^{2}\left(S_{r}, m_{r}, C^{2 j+1}\right), m_{r}$ the Lebesgue measure on the sphere of radius $e^{r}$, defined by

$$
W(r)(t, R) f(s)=e^{2 \pi i x \cdot s} D^{j}(R) f\left(R^{-1} y\right) .
$$

One can show $W(r)=\operatorname{ind}_{K}^{M}(W \cdot t)=\operatorname{ind}_{t K t^{-1}}^{M} W \cdot t$ where $W(x, R)=$ $e^{2 \pi i x_{1}} D^{j}(R)$ for $\left(x_{1}, x_{2}, x_{3}, R\right) \in K$. Hence $U=\operatorname{ind}_{K}^{G} W$.

For more information on particle systems having these symmetries, see [1]. 


\section{REFERENCES}

1. R. Fabec, Free particle systems and their dynamics in de Sitter space, J. Mathematical Phys. 18 (1977), 2087-2091.

2. G. W. Mackey, Induced representations of a locally compact group. I, Ann. of Math. 2 (1952), 101-139.

3. The theory of unitary group representations, 1st ed., Univ. of Chicago Press, Chicago, III., 1976, 372 pp.

4. __, Unitary representations of group extensions. I, Acta Math. 99 (1958), 265-311.

Departmant of Mathematics, Louisiana State University, Baton Rouge, Louisiana 70803 